\title{
Estimations for spectral radius of nonnegative matrices and the smallest eigenvalue of $M$-matrices
}

Te Wang, Hongbin LV $v^{*}$ and Haifeng Sang

"Correspondence: hbinlv@126.com Mathematics Department, Beihua University, Jilin, 132033, China

\begin{abstract}
In this paper, some estimations for the spectral radius of nonnegative matrices and the smallest eigenvalue of $M$-matrices are given by matrix directed graphs and their $k$-path covering. The existent results on the upper and lower bounds of the spectral radius of nonnegative matrices are improved.
\end{abstract}

MSC: $15 \mathrm{~A} 18 ; 65 \mathrm{~F} 15$

Keywords: nonnegative matrix; $M$-matrix; spectral radius; eigenvalues

\section{Introduction}

The following notations are used throughout this paper. Let $A=\left(a_{i j}\right) \in \mathbb{R}^{n \times n}$ be an $n \times n$ matrix with real entries. Denote $\mathcal{N}=\{1,2, \ldots, n\}, r_{i}(A)=\sum_{j \in \mathcal{N}}\left|a_{i j}\right|, R_{i}(A)=\sum_{j \in \mathcal{N} \backslash\{i\}}\left|a_{i j}\right|$, $i \in \mathcal{N} . A[\mathcal{P}]$ denotes the prime sub-matrices of $A$ where row-column subscripts are all in $\mathcal{P} \subseteq \mathcal{N}, \rho(A)$ is the spectral radius of $A$, and $\omega_{0}(A)$ is the smallest eigenvalue according to the module of $A$.

For $A=\left(a_{i j}\right) \in \mathbb{R}^{n \times n}$, if $a_{i j} \geq 0, i, j \in \mathcal{N}, A$ is called a nonnegative matrix. We denote it $A \in \mathcal{N}_{n}$. If $A=s I-B, B \in \mathcal{N}_{n}, s>\rho(B), A$ is called a nonsingular $M$-matrix. We denote it $A \in K$. Nonnegative matrices and $M$-matrices are two important matrix classes, which are applied in many fields such as computational mathematics, probability theory, and mathematical economics (see [1]). Some spectral properties of these two classes of matrices are discussed in the paper.

With regard to estimations for the nonnegative matrix spectral radius, the earliest result is given by Perron-Frobenius (see [2]), that is,

$$
\min _{i \in \mathcal{N}} r_{i}(A) \leq \rho(A) \leq \max _{i \in \mathcal{N}} r_{i}(A)
$$

Though the result is earlier than the Geršgorin theorem (see [3]), it can be seen as the estimation of $\rho(A)$ by using the right end-point of the Geršgorin disc. Therefore it is still called a Geršgorin estimation.

Denote

$$
r_{A}(i, j)=\frac{1}{2}\left\{a_{i i}+a_{j j}+\left[\left(a_{i i}-a_{j j}\right)^{2}+4 R_{i}(A) R_{j}(A)\right]^{\frac{1}{2}}\right\}, \quad i \neq j, \forall i, j \in \mathcal{N} .
$$

O2014 Wang et al.; licensee Springer. This is an Open Access article distributed under the terms of the Creative Commons Attribution License (http://creativecommons.org/licenses/by/2.0), which permits unrestricted use, distribution, and reproduction in any medium, provided the original work is properly cited. 
A Brauer (see [4]) gave the Brauer estimation for the spectral radius of nonnegative matrices by the Cassini oval region, and he improved Perron-Frobenius' result.

Suppose $A \in \mathcal{N}_{n}$ is inducible, then

$$
\min _{i \neq j}\left\{r_{A}(i, j)\right\} \leq \rho(A) \leq \max _{i \neq j}\left\{r_{A}(i, j)\right\} .
$$

A main equivalent representation of $M$-matrices indicates that the real parts of $M$ matrix eigenvalues are all positive (see [1]). Tong (see [5]) improved the result and concluded that the min-eigenvalue by the module of an $M$-matrix is a positive number. Zhang (see [6]) proved that the min-eigenvalue of an $M$-matrix by its real part is also its mineigenvalue by the module and offered the estimation formula

$$
\min _{i \in \mathcal{N}} r_{i}(A) \leq \omega_{0}(A) \leq \max _{i \in \mathcal{N}} r_{i}(A)
$$

For the same reason, we also call it a Geršgorin type estimation. Let $A$ be a nonsingular $M$-matrix and denote $s=\max _{i \in \mathcal{N}}\left\{a_{i i}\right\}$. Then $A=s I-B, B \in \mathcal{N}_{n}$, thus $\omega(A)=s-\rho(B)$, and the above estimation results as regards the $M$-matrix are natural. Therefore, according to [4], we can give the Brauer estimation for the $M$-matrix min-eigenvalues.

Denote

$$
l_{A}(i, j)=\frac{1}{2}\left\{a_{i i}+a_{j j}-\left[\left(a_{i i}-a_{j j}\right)^{2}+4 R_{i}(A) R_{j}(A)\right]^{\frac{1}{2}}\right\}, \quad i \neq j, \forall i, j \in \mathcal{N} .
$$

Let $A$ be a nonsingular and irreducible $M$-matrix. Then

$$
\min _{i \neq j}\left\{l_{A}(i, j)\right\} \leq \omega(A) \leq \max _{i \neq j}\left\{l_{A}(i, j)\right\} .
$$

In this paper, we present the Brualdi type estimation for the nonnegative matrix spectral radius and the minimal eigenvalue of $M$-matrix by the deduction method with directed graph (see [7]) and the concept of the $k$-path covering of the directed graph. Moreover, we give the improved Brauer type estimations, which improve the relevant results of [4-6, $8-10]$.

\section{Directed graph and its $k$-path covering}

Let $\Gamma(A)$ be the directed graph of $A=\left(a_{i j}\right) \in \mathbb{C}^{n \times n}$. $\mathcal{N}$ denotes its nodal set, and $E(A)=$ $\left\{e_{i, j} \mid a_{i j} \neq 0, i, j \in \mathcal{N}\right\}$ denotes its directed edge set. The directed edge sequence $\gamma$ : $e_{i_{1}, i_{2}}, e_{i_{2}, i_{3}}, \ldots, e_{i_{s-1}, i_{s}}, e_{i_{s}, i_{1}}$ is called the simple loop of $\Gamma(A)$ where $s \geq 2$ and all $i_{1}, i_{2}, \ldots, i_{s}$ are different from each other. In short, we denote $\gamma: i_{1}, i_{2}, \ldots, i_{s}, i_{s+1}=i_{1}$. Let $|\gamma|$ be the length of the simple loop $\gamma \in C(A)$, then $C(A)$ is the set of all the simple loops of matrix $A$.

In this paper, directed graphs and simple loops of $\Gamma(A)$ are all regarded as its sub-graphs, and $\Gamma^{+}(i)=\left\{j \in \mathcal{N} \mid j \neq i, e_{i, j} \in E(A)\right\}$ means $i$ 's successor set in $\Gamma(A)$.

We will introduce the concept of $k$-path coverage of matrix directed graphs (see [11]).

Definition $2.1[11]$ Let $\gamma: i_{1}, i_{2}, \ldots, i_{s}, i_{s+1}=i_{1} \in C(A), k$ be an integer, $t=\min \{k+1, s\}$, and $[t, s]=p t=q s$ be the least common multiple of $t$ and $s$. We call the set $\left\{\rho_{1}, \ldots, \rho_{p}\right\}$ made up of directed paths in $\gamma$, respectively, starting with $i_{j}, i_{j+t}, \ldots, i_{j+(p-1) t}$, and including $t-1$ directed edges as the $k$-path coverage of the simple loop $\gamma$ ( $i_{j}$ as base point), denoted by $P^{k}(\gamma)$. 
Definition 2.2 [11] For every $\gamma \in C(A)$ in $\Gamma(A), P^{k}(A)=\bigcup_{\gamma \in C(A)} P^{k}(\gamma)$ is called a $k$-path coverage in $\Gamma(A)$.

Obviously, if the chosen base-points are different, usually there are different $k$-path coverings $P^{k}(\gamma)$. When $k=l|\gamma|, l \in \mathbb{Z}^{+}$, the $k$-path coverage on $\gamma \in C(A)$ is $\gamma$ itself. If $|\gamma|=p(k+1), \gamma \in C(A)$, then $P^{k}(\gamma)$ only covers every nodal point on $\gamma$ once and there are $k+1$ different $k$-path coverings $P^{k}(\gamma)$ on $\gamma$. If $k \geq \max _{\gamma \in C(A)}|\gamma|$, then $P^{k}(A)=C(A)$.

Besides, when $k=1$, let $\gamma: i_{1}, i_{2}, \ldots, i_{s}, i_{s+1}=i_{1} \in C(A), \eta$ be the maximal common divisor of 2 and $s$, and $\tau=s / \eta$. The set $\left\{e_{i_{1}, i_{2}}, e_{i_{1+\eta}, i_{2+\eta}}, \ldots, e_{i_{1+(\tau-1) \eta}, i_{2+(\tau-1) \eta}}\right\}$ is called an odd 1-path covering of $\gamma$ (corresponding to the contemporary notation); and the set $\left\{e_{i_{2}, i_{3}}\right.$, $\left.e_{i_{2+\eta}, i_{3+\eta}}, \ldots, e_{i_{2+(\tau-1) \eta}, i_{3+(\tau-1) \eta}}\right\}$ is called an even 1-path coverage of $\gamma$ (corresponding to the contemporary notation). Likewise, a definitive 1-path coverage of $\gamma$ is denoted as $P^{1}(\gamma)$. When $s$ is a positive odd number, the even 1-path and the odd one of $\gamma$ are the same. Namely there is only a 1-path coverage including all $s$ directed edges in $\gamma$. When $s$ is a positive even number, there are two odd and even 1-path coverings, respectively, including its $s / 2$ pieces of directed edges in $\gamma$.

A relation ' $\prec$ ' on the nonempty set $v$ is called pre-order, if ' $\prec$ ' satisfies reflexivity and transitivity, namely $a \prec a, \forall a \in v ; a \prec b$ and $b \prec c$ implies $a \prec c, a, b, c \in v$.

Lemma $2.1[7,11]$ Let ' $\prec$ ' be a pre-order on the nodal set $\mathcal{N}=\{1,2, \ldots, n\}$ of $\Gamma(A), n \geq 2$. If for all $i \in \mathcal{N}, \Gamma^{+}(i) \neq \emptyset$, then:

(1) If for all $i \in \mathcal{N}, \Gamma^{+}(i) \neq \emptyset$, then there exists a simple loop $\gamma: i_{1}, i_{2}, \ldots, i_{s}, i_{s+1}=i_{1}$ such that

$$
l \prec i_{j+1}, \quad \forall l \in \Gamma^{+}\left(i_{j}\right), j=1,2, \ldots, s .
$$

(2) If $P^{k}(\gamma)$ is a $k$-path covering of the above simple loop, then there exists a directed path $\rho: m_{1}, m_{2}, \ldots, m_{t} \in P^{k}(\gamma)$ in $\gamma$, such that

$$
\begin{aligned}
& l \prec m_{j+1}, \quad \forall l \in \Gamma^{+}\left(m_{j}\right), j=1,2, \ldots, t, \\
& m_{t+1} \prec m_{1} .
\end{aligned}
$$

Proof (1) See [7].

(2) Without loss of generality, we suppose that $i_{1}$ is the base-point of $P^{k}(\gamma)=\left(\rho_{1}, \ldots, \rho_{p}\right)$, $t=\min \{k+1, s\},[t, s]=p t=q s$, and $\rho_{j}: i_{1+(j-1) t}, i_{2+(j-1) t}, \ldots, i_{j t}$ for $1 \leq j \leq p$. Let $m_{1}$ be the largest element of the nodal set $\left\{i_{1}, i_{1+t}, \ldots, i_{1+(p-1) t}\right\}$ in the sense of pre-order ' $\prec$ '. In $\gamma$, take a directed path $\rho: m_{1}, m_{2}, \ldots, m_{t}$ starting with $m_{1}$ including $t-1$ directed edges, then $\rho \in P^{k}(\gamma)$. According to (2.1), then (2.2) holds. Because $m_{1}$ is the largest element of $\left\{i_{1}, i_{1+t}, \ldots, i_{1+(p-1) t}\right\}$ and $m_{t+1} \in\left\{i_{1}, i_{1+t}, \ldots, i_{1+(p-1) t}\right\},(2.3)$ holds.

The discussion in this paper needs some basic results as regards a reducible matrix determined by a polynomial.

If $A$ is a reducible matrix with $n \geq 2$, then there exists a permutation matrix $P$ such that

$$
P A P^{\mathrm{T}}=\left[\begin{array}{cccc}
A_{11} & A_{12} & \cdots & A_{1 K} \\
0 & A_{22} & \cdots & A_{2 K} \\
\vdots & \ddots & \ddots & \vdots \\
0 & \cdots & 0 & A_{K K}
\end{array}\right],
$$


where $A_{t t}$ is $n_{t} \times n_{t}$ principal sub-matrix of $A$ and $2 \leq K \leq n$. They are either irreducible or zero matrix with order $1, \sum_{t=1}^{K} n_{t}=n$. The right-side matrix of (2.4) is called the reduced polynomial of $A$. If the order of $A_{t t}$ is not considered, (2.4) has nothing to do with the choice of $P$. So (2.4) is a unique definite partition $\mathcal{N}_{1}, \mathcal{N}_{2}, \ldots, \mathcal{N}_{K}$ on a set $\mathcal{N}=\{1,2, \ldots, n\}$ corresponding to the subscripted set of $A_{11}, A_{22}, \ldots, A_{K K}$. When $A$ is an irreducible matrix and a zero matrix with order 1 , for unity, denote $A=\left[A_{11}\right]$, where $n_{1}=n$ and $\mathcal{N}_{1}=\mathcal{N}$.

Denote

$$
\alpha=\bigcup_{n_{t} \geq 2,1 \leq t \leq K} \mathcal{N}_{t}, \theta_{A}=\left\{a_{i i} A \mid i \in \mathcal{N} \backslash \alpha\right\} .
$$

Obviously $\alpha=\{i \in \mathcal{N} \mid i \in \gamma \in C(A)\}$.

Definition 2.3 Let $A=\left(a_{i j}\right) \in \mathbb{C}^{n \times n}$. If $\mathcal{N}=\alpha$, then $A$ is a weakly irreducible matrix, which is denoted by $A \in W I$. For a general matrix, if $\alpha \neq \emptyset$, we call $A[\alpha]$ the weakly irreducible nucleus of $A$, which is denoted by $\hat{A}$. Denote $\hat{A}=\emptyset$ if $\alpha=\emptyset$.

\section{The spectral radius of a nonnegative matrix}

In this section, we suppose that $\max \emptyset=\min \emptyset=0$.

Lemma 3.1 Let $a_{1}, a_{2}, \ldots, a_{n} \in \mathbb{R}, \mathcal{N}=\{1,2, \ldots, n\}, \mathcal{J} \subseteq \mathcal{N}$. Define a function $f(x)=$ $\prod_{i \in \mathcal{J}}\left(x-a_{i}\right)$. Then $f(x)$ is strongly monotone increasing when $x \geq \max _{i \in \mathcal{J}}\left\{a_{i}\right\}$.

Theorem 3.1 Let $A=\left(a_{i j}\right) \in \mathcal{N}_{n} . \forall \gamma \in C(A), r_{A}(\gamma)$ denotes the real roots of $\prod_{i \in \gamma}\left(x-a_{i i}\right)=$ $\prod_{i \in \gamma} R_{i}(\hat{A})$ which are larger than $\max _{i \in \gamma}\left\{a_{i i}\right\}$. Denote

$$
\begin{aligned}
& m_{c}^{r}(A)=\max \left\{\min _{\gamma \in C(A)} r_{A}(\gamma), \max \Theta_{A}\right\}, \\
& M_{c}^{r}(A)=\max \left\{\max _{\gamma \in C(A)} r_{A}(\gamma), \max \Theta_{A}\right\},
\end{aligned}
$$

then

$$
m_{c}^{r}(A) \leq \rho(A) \leq M_{c}^{r}(A)
$$

Proof (1) $A$ is irreducible. By the Perron-Frobenius theorem, we know there is $x=$ $\left(x_{1}, x_{2}, \ldots, x_{n}\right)^{\mathrm{T}}>0$, such that $A x=\rho(A) x$. Define the pre-order ' $\prec$ ': $i \prec j$ on the nodal set $\mathcal{N}$ of $\Gamma(A)$, if and only if $x_{i} \geq x_{j}$. From Lemma 2.1(1), there exists $\gamma^{\prime}: i_{1}, i_{2}, \ldots, i_{s}, i_{s+1}=i_{1} \in C(A)$ such that $x_{l} \geq x_{i_{j+1}}, \forall l \in \Gamma^{+}\left(i_{j}\right), j=1,2, \ldots, s$. Hence, from

$$
\begin{aligned}
\left(\rho(A)-a_{i_{j} i_{j}}\right) x_{i_{j}} & =\sum_{p \neq i_{j}} a_{i_{j} p} x_{p}=\sum_{p \in \Gamma^{+}\left(i_{j}\right)} a_{i_{j} p} x_{p} \\
& \geq\left(\sum_{p \in \Gamma^{+}\left(i_{j}\right)} a_{i_{j} p}\right) x_{i_{j+1}}=R_{i_{j}}(A) x_{i_{j+1}}, \quad j=1,2, \ldots, s,
\end{aligned}
$$

we obtain

$$
\prod_{j=1}^{s}\left(\rho(A)-a_{i_{j} i_{j}}\right) \prod_{j=1}^{s} x_{i_{j}} \geq \prod_{j=1}^{s} R_{i_{j}}(A) \prod_{j=1}^{s} x_{i_{j+1}} .
$$


Then

$$
\prod_{j=1}^{s}\left(\rho(A)-a_{i_{j} i_{j}}\right) \geq \prod_{j=1}^{s} R_{i_{j}}(A)
$$

i.e.

$$
\prod_{i \in \gamma^{\prime}}\left(\rho(A)-a_{i i}\right) \geq \prod_{i \in \gamma^{\prime}} R_{i}(A) .
$$

Similarly, if we define a pre-order ' $\prec$ ': $i \prec j$ on the nodal set $\mathcal{N}$ of $\Gamma(A)$, if and only if $x_{i} \leq x_{j}$. From Lemma 2.1, there exists $\gamma^{\prime \prime}: i_{1}, i_{2}, \ldots, i_{s}, i_{s+1}=i_{1} \in C(A)$ such that $x_{l} \leq x_{i_{j+1}}$, $\forall l \in \Gamma^{+}\left(i_{j}\right), j=1,2, \ldots, s$. Similar to the above, we obtain

$$
\prod_{i \in \gamma^{\prime \prime}}\left(\rho(A)-a_{i i}\right) \leq \prod_{i \in \gamma^{\prime \prime}} R_{i}(A) .
$$

Besides, notice $\rho(A) \geq \max _{i \in \gamma^{\prime}}\left\{a_{i i}\right\}$ and $\rho(A) \geq \max _{i \in \gamma^{\prime \prime}}\left\{a_{i i}\right\}$. From (3.1) and (3.2), it is deduced that

$$
\min _{\gamma \in C(A)} r_{A}(\gamma) \leq r_{A}\left(\gamma^{\prime}\right) \leq \rho(A) \leq r_{A}\left(\gamma^{\prime \prime}\right) \leq \max _{\gamma \in C(A)} r_{A}(\gamma)
$$

i.e. $m_{c}^{r}(A) \leq \rho(A) \leq M_{c}^{r}(A)$.

(2) $A$ is weakly irreducible. It can be supposed that $A$ has got its polynomial (2.4), where $A_{t t}$ is irreducible whose order is not less than 2 . We prove it with the following two steps.

(i) Since $R_{i}(\hat{A})=R_{i}\left(A_{K K}\right), \forall i \in \mathcal{N}_{K}$. From (1), we easily see

$$
\rho(A) \geq \rho\left(A_{K K}\right) \geq m_{c}^{r}\left(A_{K K}\right)=\min _{\gamma \in C\left(A_{K K}\right)} r_{A_{K K}}(\gamma)=\min _{\gamma \in C\left(A_{K K}\right)} r_{A}(\gamma) \geq \min _{\gamma \in C(A)} r_{A}(\gamma) .
$$

(ii) Let $t^{*}$ such that $\rho(A)=\rho\left(A_{t^{*} t^{*}}\right)$. From (1), we easily see that

$$
\rho(A)=\rho\left(A_{t^{*} t^{*}}\right) \leq M_{c}^{r}\left(A_{t^{*} t^{*}}\right)=\max _{\gamma \in C\left(A_{t^{*} t^{*}}\right)} r_{A_{t^{*} t^{*}}}(\gamma) \leq \max _{\gamma \in C\left(A_{t^{*} t^{*}}\right)} r_{A}(\gamma) \leq \max _{\gamma \in C(A)} r_{A}(\gamma) .
$$

Combining (i) with (ii), we have $m_{c}^{r}(A) \leq \rho(A) \leq M_{c}^{r}(A)$.

(3) $A$ is weakly irreducible. Noticing $r_{A}(\gamma)=r_{\hat{A}}(\gamma)$, from (2), we see

$$
\min _{\gamma \in C(A)} r_{A}(\gamma)=\min _{\gamma \in C(\hat{A})} r_{\hat{A}}(\gamma) \leq \rho(\hat{A}) \leq \max _{\gamma \in C(\hat{A})} r_{\hat{A}}(\gamma)=\max _{\gamma \in C(A)} r_{A}(\gamma)
$$

Since

$$
\begin{aligned}
& \rho(A)=\max \left\{\max \Theta_{A}, \rho(\hat{A})\right\}, \\
& \max \left\{\min _{\gamma \in C(A)} r_{A}(\gamma), \max \Theta_{A}\right\} \leq \rho(A) \leq \max \left\{\max _{\gamma \in C(A)} r_{A}(\gamma), \max \Theta_{A}\right\},
\end{aligned}
$$

i.e. $m_{c}^{r}(A) \leq \rho(A) \leq M_{c}^{r}(A)$. 
Remark 3.1 Because $m_{c}^{r}(A)$ and $M_{c}^{r}(A)$ have relations to the directed graphs, when $A$ is reducible, and traditional continuity deduction has no effect, we use (2.4) to prove it. Besides, in Theorem 3.1, $r_{A}(\gamma)$ must be defined by $R_{i}(\hat{A})$, but it cannot be defined by $R_{i}(A)$ directly. See Example 5.3.

Theorem 3.2 Let $A=\left(a_{i j}\right) \in \mathcal{N}_{n}$ and $P^{k}(A)$ be the k-path covering of $\Gamma(A) . \forall \rho \in P^{k}(A)$, always denote its nodal set as $\rho: i_{1}, i_{2}, \ldots, i_{k}, i_{k+1} . r_{A}(\rho)$ denotes the real roots of the equation $\prod_{i \in \rho}\left(x-a_{i i}\right)=\prod_{i \in \rho} R_{i}(\hat{A}), r_{A}(\rho)>\max _{i \in \rho}\left\{a_{i i}\right\}$. Denote

$$
\begin{aligned}
& m_{\rho}^{r}(A)=\max \left\{\min _{\rho \in P^{k}(A)} r_{A}(\rho), \max \Theta_{A}\right\}, \\
& M_{\rho}^{r}(A)=\max \left\{\max _{\rho \in P^{k}(A)} r_{A}(\rho), \max \Theta_{A}\right\},
\end{aligned}
$$

then

$$
m_{\rho}^{r}(A) \leq \rho(A) \leq M_{\rho}^{r}(A)
$$

Proof Let $A$ be an irreducible and nonnegative matrix. By the Perron-Frobenius theorem, there exists $x=\left(x_{1}, \ldots, x_{n}\right)^{\mathrm{T}}>0$ such that $A x=\rho(A) x$. Define a pre-order ' $\prec$ ': $i \prec j$ on the nodal set $\mathcal{N}$ of $\Gamma(A)$, if and only if $x_{i} \leq x_{j}$. It follows from Lemma 2.1 that there exists a directed path $\rho^{\prime}: m_{1}, m_{2}, \ldots, m_{t} \in P^{k}(A)$ in $\Gamma(A)$, such that $x_{l} \leq x_{j+1}, \forall l \in \Gamma^{+}\left(m_{j}\right), j=1, \ldots, t$; $x_{m_{t+1}} \leq x_{m_{1}} ; x_{m_{j}}>0, j=1,2, \ldots, t$. Because $A x=\rho(A) x$, we get

$$
\begin{aligned}
0 & <\left(\rho(A)-a_{m_{j} m_{j}}\right) x_{m_{j}}=\sum_{p \neq m_{j}} a_{m_{j} p} x_{p} \\
& =\sum_{p \in \Gamma^{+}\left(m_{j}\right)} a_{m_{j} p} x_{p} \leq\left(\sum_{p \in \Gamma^{+}\left(m_{j}\right)} a_{m_{j} p}\right) x_{m_{j+1}} \\
& =\left(\sum_{p \neq m_{j}} a_{m_{j} p}\right) x_{m_{j+1}}=R_{m_{j}}(A) x_{m_{j+1}}, \quad j=1,2, \ldots, t .
\end{aligned}
$$

Multiply all the inequalities in (3.3):

$$
\prod_{j=1}^{t}\left(\rho(A)-a_{m_{j} m_{j}}\right) \prod_{j=1}^{t} x_{m_{j}} \leq \prod_{j=1}^{t} R_{m_{j}}(A) \prod_{j=1}^{t} x_{m_{j+1}} .
$$

Furthermore,

$$
\prod_{j=1}^{t}\left(\rho(A)-a_{m_{j} m_{j}}\right) \leq\left(\prod_{j=1}^{t} R_{m_{j}}(A)\right) \frac{x_{m_{t+1}}}{x_{m_{1}}} .
$$

Then

$$
\prod_{i \in \rho^{\prime}}\left(\rho(A)-a_{m_{j} m_{j}}\right) \leq \prod_{i \in \rho^{\prime}} R_{i}(A)
$$


Likewise, a pre-order ' $\prec$ ': $i \prec j$ is defined on the nodal set $\mathcal{N}$ of $\Gamma(A)$ if and only if $x_{i} \geq x_{j}$. It follows from Lemma 2.1 that there exists a directed path $\rho^{\prime \prime}: m_{1}, m_{2}, \ldots, m_{t} \in P^{k}(A)$ in $\Gamma(A)$ such that $x_{l} \geq x_{j+1}, \forall l \in \Gamma^{+}\left(m_{j}\right), j=1,2, \ldots, t ; x_{m_{t+1}} \geq x_{m_{1}}$, and $x_{m_{j}}>0, j=1,2, \ldots, t$. Therefore from $A x=\rho(A) x$, we obtain

$$
\prod_{i \in \rho^{\prime \prime}}\left(\rho(A)-a_{m_{j} m_{j}}\right) \geq \prod_{i \in \rho^{\prime \prime}} R_{i}(A)
$$

Thus, if $A \in \mathcal{N}_{n}$ is irreducible, with (3.4) and (3.5), the theorem is proved. If $A \in \mathcal{N}_{n}$ is a weakly irreducible or non-weakly irreducible matrix, similar to the proof of (2), (3) in Theorem 3.1, it is the same with the theorem here.

In Theorem 3.2, if $k \geq \max _{\gamma \in C(A)}|\gamma|$, it is Theorem 3.1. Therefore Theorem 3.1 can be viewed as a special case of Theorem 3.2.

Theorem 3.3 Let $A=\left(a_{i j}\right) \in \mathcal{N}_{n}$, and $P^{k}(A)$ be the k-path covering of $\Gamma(A), \forall \rho \in P^{k}(A)$, its nodal set is always denoted as $\rho: i_{1}, i_{2}, \ldots, i_{k}, i_{k+1}$. The other notations are the same as those in Theorem 3.1 and Theorem 3.2. Then

$$
m_{\rho}^{r}(A) \leq m_{c}^{r}(A) \leq \rho(A) \leq M_{c}^{r}(A) \leq M_{\rho}^{r}(A)
$$

Proof First we prove $m_{\rho}^{r}(A) \leq m_{c}^{r}(A)$. It is necessary to prove that for all $\gamma \in C(A)$ there exists $\rho \in P^{k}(\gamma) \subseteq P^{k}(A)$ such that $r_{A}(\gamma) \geq r_{A}(\rho)$. Otherwise there exists $\gamma: i_{1}, i_{2}, \ldots, i_{s}, i_{s+1}=$ $i_{1} \in C(A),|\gamma|=s$, such that $r_{A}(\gamma)<r_{A}(\rho), \forall \rho \in P^{k}(\gamma)$. Note that $r_{A}(\rho)$ is the real root of the equation $\prod_{i \in \rho}\left(x-a_{i i}\right)=\prod_{i \in \rho} R_{i}(\hat{A})$, larger than $\max _{i \in \rho}\left\{a_{i i}\right\}$. From Lemma 3.1, we see that

$$
\prod_{i \in \rho}\left(r_{A}(\gamma)-a_{i i}\right)<\prod_{i \in \rho} R_{i}(\hat{A}), \quad \forall \rho \in P^{k}(\gamma) .
$$

Multiply all the inequalities in (3.6),

$$
\prod_{\rho \in P^{k}(\gamma)}\left(\prod_{i \in \rho}\left(r_{A}(\gamma)-a_{i i}\right)\right)<\prod_{\rho \in P^{k}(\gamma)}\left(\prod_{i \in \rho} R_{i}(\hat{A})\right),
$$

i.e.

$$
\left(\prod_{i \in \gamma}\left(r_{A}(\gamma)-a_{i i}\right)\right)^{p(k+1) /|\gamma|}<\left(\prod_{i \in \gamma} R_{i}(\hat{A})\right)^{p(k+1) /|\gamma|} .
$$

Furthermore

$$
\prod_{i \in \gamma}\left(r_{A}(\gamma)-a_{i i}\right)<\prod_{i \in \gamma} R_{i}(\hat{A})
$$

Then from Lemma 3.1, we know that (3.7) implies $r_{A}(\gamma)<r_{A}(\gamma)$, which leads to a contradiction. 
Similarly, $M_{c}^{r}(A) \leq M_{\rho}^{r}(A)$ can be proved. With the above and Theorem 3.1, the theorem is proved.

If $k=1$, the following result, which is more convenient to use, can be obtained from Theorem 3.2.

Corollary 3.1 Let $A=\left(a_{i j}\right) \in \mathcal{N}_{n}$, and $P^{1}(A)$ be the 1-path covering of $\Gamma(A)$. Denote

$$
\begin{aligned}
& r_{A}(i, j)=\frac{1}{2}\left\{a_{i i}+a_{i j}+\left[\left(a_{i i}-a_{j j}\right)^{2}+4 R_{i}(\hat{A}) R_{j}(\hat{A})\right]^{\frac{1}{2}}\right\}, \\
& m_{e}^{r}(A)=\max \left\{\min _{e_{i, j} \in P^{1}(A)} r_{A}(i, j), \max \Theta_{A}\right\}, \\
& M_{e}^{r}(A)=\max \left\{\max _{e_{i, j} \in P^{1}(A)} r_{A}(i, j), \max \Theta_{A}\right\} .
\end{aligned}
$$

Then

$$
m_{e}^{r}(A) \leq \rho(A) \leq M_{e}^{r}(A)
$$

Obviously, generally speaking, different $k$-path coverings can be taken for the directed graph $\Gamma(A)$ of $A \in \mathbb{C}^{n \times n}$. We denote the congregation set of these different $k$-path coverings $P^{k}(A)$ of $\Gamma(A)$ as $\bigcup P^{k}(A)$, then we have the following theorem.

Theorem 3.4 Let $A=\left(a_{i j}\right) \in \mathcal{N}_{n}$, for a given k-path covering $P^{k}(A) \in \bigcup P^{k}(A)$ of $\Gamma(A)$, with $M_{\rho}^{r}(A), m_{\rho}^{r}(A)$, the same as in Theorem 3.2. Then

$$
\max _{P^{k}(A) \in \bigcup P^{k}(A)}\left\{m_{\rho}^{r}(A)\right\} \leq \rho(A) \leq \min _{P^{k}(A) \in \bigcup P^{k}(A)}\left\{M_{\rho}^{r}(A)\right\} .
$$

Remark 3.2 Theorem 3.1 can be viewed as the estimation for $\rho(A)$ by means of the right end-point of the Brualdi region of an eigenvalue distribution, so it is called a Brualdi estimation. Corollary 3.1 is the improved Brauer estimation. Because we only need to calculate the corresponding $r_{A}(i, j)$ of the edge $e_{i, j}$ in the simple loop, especially when the length of the loop is even, only the corresponding $r_{A}(i, j)$ of half of the edges needs to be calculated. Thus the calculation decreases greatly and meanwhile the accuracy improves. If Theorem 3.4 is used, a general estimation is more accurate. Theorem 3.1 and Corollary 3.1 are both superior to the results of Perron-Frobenius and Brauer. See Example 5.1 and Example 5.2.

\section{The smallest eigenvalue of $M$-matrix}

In this section, we define $\max \emptyset=\min \emptyset=+\infty$.

Theorem 4.1 Let $A=\left(a_{i j}\right) \in \mathbb{R}^{n \times n}$ be a nonsingular M-matrix. $\forall \gamma \in C(A), l_{A}(\gamma)$ is the real root of the equation $\prod_{i \in \gamma}\left(a_{i i}-x\right)=\prod_{i \in \gamma} R_{i}(\hat{A})$, and $l_{A}(\gamma)<\min _{i \in \gamma}\left\{a_{i i}\right\}$. Denote

$$
\begin{aligned}
& m_{c}^{l}(A)=\min \left\{\min _{\gamma \in C(A)} l_{A}(\gamma), \min \Theta_{A}\right\}, \\
& M_{c}^{l}(A)=\min \left\{\max _{\gamma \in C(A)} l_{A}(\gamma), \min \Theta_{A}\right\} .
\end{aligned}
$$


Then

$$
m_{c}^{l}(A) \leq \omega_{0}(A) \leq M_{c}^{l}(A) .
$$

Proof Let $A=s I-B, B=\left(b_{i j}\right) \in \mathcal{N}_{n}, \rho(B)$ be the spectral radius of $B$ and $s>\rho(B)$. Obviously $\omega_{0}(A)=s-\rho(B)>0$. It follows from Theorem 3.1 that $m_{c}^{r}(B) \leq \rho(B) \leq M_{c}^{r}(B)$, and then $s-M_{c}^{r}(B) \leq \omega_{0}(A) \leq s-m_{c}^{r}(B)$.

Define $a_{i i}=s-b_{i i}, i \in \mathcal{N}$. Then it follows from the definitions of $l_{A}(\gamma)$ and $r_{A}(\gamma)$ that $l_{A}(\gamma)=s-r_{B}(\gamma)$. We obtain

$$
\begin{aligned}
m_{c}^{l}(A) & =\min \left\{\min _{\gamma \in C(A)} l_{A}(\gamma), \min \Theta_{A}\right\} \\
& =\min \left\{\min _{\gamma \in C(B)}\left\{s-r_{B}(\gamma)\right\}, s-\max \Theta_{B}\right\} \\
& =s-\max \left\{\max _{\gamma \in C(B)} r_{B}(\gamma), \max \Theta_{B}\right\} \\
& =s-M_{c}^{r}(B) .
\end{aligned}
$$

Similarly, it follows that $M_{c}^{l}(A)=s-m_{c}^{r}(B)$. So $m_{c}^{l}(A) \leq \omega_{0}(A) \leq M_{c}^{l}(A)$.

Analogously, we have the following results.

Theorem 4.2 Let $A=\left(a_{i j}\right) \in \mathbb{R}^{n \times n}$ be a nonsingular M-matrix, and $P^{k}(A)$ be the $k$-path covering of $\Gamma(A) . \forall \rho \in P^{k}(A)$, always denote its nodal set as $\rho: i_{1}, i_{2}, \ldots, i_{k}, i_{k+1}$. Denoted by $r_{A}(\rho)$ the real root of the equation $\prod_{i \in \rho}\left(a_{i i}-x\right)=\prod_{i \in \rho} R_{i}(\hat{A})$, which is less than $\min _{i \in \rho}\left\{a_{i i}\right\}$, and denote

$$
\begin{aligned}
& m_{\rho}^{l}(A)=\min \left\{\min _{\rho \in P^{k}(A)} l_{A}(\rho), \min \Theta_{A}\right\}, \\
& M_{\rho}^{l}(A)=\min \left\{\max _{\rho \in P^{k}(A)} l_{A}(\rho), \min \Theta_{A}\right\} .
\end{aligned}
$$

Then

$$
m_{\rho}^{l}(A) \leq \omega_{0}(A) \leq M_{\rho}^{l}(A)
$$

and

$$
m_{\rho}^{l}(A) \leq m_{c}(A) \leq \omega_{0}(A) \leq M_{c}(A) \leq M_{\rho}^{l}(A) .
$$

If we take $k=1$, we have the following corollary.

Corollary 4.1 Let $A=\left(a_{i j}\right) \in \mathbb{R}^{n \times n}$ be a nonsingular $M$-matrix, $P^{1}(A)$ is the 1-path covering of $\Gamma(A)$. Denote

$$
\begin{aligned}
& l_{A}(i, j)=\frac{1}{2}\left\{a_{i i}+a_{j j}-\left[\left(a_{i i}-a_{j j}\right)^{2}+4 R_{i}(\hat{A}) R_{j}(\hat{A})\right]^{\frac{1}{2}}\right\}, \\
& m_{e}^{l}(A)=\min \left\{\min _{e_{i, j} \in P^{1}(A)} l_{A}(i, j), \min \Theta_{A}\right\}, \\
& M_{e}^{l}(A)=\min \left\{\max _{e_{i, j} \in P^{1}(A)} l_{A}(i, j), \min \Theta_{A}\right\} .
\end{aligned}
$$


Then

$$
m_{e}^{l}(A) \leq \omega_{0}(A) \leq M_{e}^{l}(A)
$$

Remark 4.1 Theorem 4.1 and Corollary 4.1 are, respectively, the Brualdi type estimation and the Brauer type estimation for the least eigenvalue of the $M$-matrix. These results are more accurate. See the matrix B in Example 5.1 and Example 5.2.

\section{Examples}

Example 5.1 Consider the nonnegative matrix

$$
A=\left[\begin{array}{lllll}
8 & 1 & 0 & 0 & 0 \\
1 & 2 & 1 & 0 & 0 \\
0 & 1 & 5 & 1 & 0 \\
0 & 0 & 1 & 2 & 1 \\
0 & 0 & 0 & 1 & 8
\end{array}\right] .
$$

By calculating, $\rho(A)=8.18014$. It follows from a Geršgorin type estimation that $4 \leq$ $\rho(A) \leq 9$. Because $r_{A}(1,2)=r_{A}(1,4)=r_{A}(2,5)=r_{A}(4,5)=8.31662, r_{A}(1,5)=9, r_{A}(2,4)=4$, $r_{A}(2,3)=r_{A}(3,4)=6$, and $r_{A}(1,3)=r_{A}(3,5)=8.56155$, it follows from a Brauer type estimation that $4 \leq \rho(A) \leq 9$. Take $P^{1}(A)=\left\{e_{1,2}, e_{2,3}, e_{3,4}, e_{4,5}\right\}$, and it follows from Corollary 3.1 that $6 \leq \rho(A) \leq 8.31662$.

Consider a nonsingular $M$-matrix $B=9 I-A$. It only follows from Geršgorin type and Brauer type estimations that $0 \leq \omega_{0}(B) \leq 5$. Take $P^{1}(B)=\left\{e_{1,2}, e_{2,3}, e_{3,4}, e_{4,5}\right\}$, and it follows from Corollary 4.1 that $0.68338 \leq \omega_{0}(B) \leq 3$. But in fact $\omega_{0}(B)=0.81986$.

Example 5.2 Consider the nonnegative matrix

$$
A=\left[\begin{array}{cccc}
1 & 0.6 & 0 & 0 \\
0 & 2 & 0.6 & 0 \\
0 & 0 & 3 & 0.6 \\
0.6 & 0 & 0 & 4
\end{array}\right]
$$

By calculating, $\rho(A)=4.02080$. It follows from a Geršgorin type estimation that $1.6 \leq$ $\rho(A) \leq 4.6$. Because $r_{A}(1,2)=2.28102, r_{A}(1,3)=3.16619, r_{A}(1,4)=4.11555, r_{A}(2,3)=$ $3.28102, r_{A}(2,4)=4.16619$, and $r_{A}(3,4)=4.28102$, it follows from a Brauer type estimation that $2.28102 \leq \rho(A) \leq 4.28102$. Take $P^{1}(A)=\left\{e_{2,3}, e_{4,1}\right\}$, and it follows from Corollary 3.1 that $3.28102 \leq \rho(A) \leq 4.11555$. Because $C(A)=\{\gamma: 1,2,3,4,1\}, m_{c}^{r}(A)=M_{c}^{r}(A)=4.02080$ and it follows from Theorem 3.1 that the estimation $\rho(A)=4.02080$ is already accurate.

Consider the nonsingular $M$-matrix $B=5 I-A$. It follows from a Geršgorin type estimation that $0.4 \leq \omega_{0}(B) \leq 3.4$ and it follows from a Brauer type estimation that $0.71898 \leq \omega_{0}(B) \leq 2.71898$. Take $P^{1}(B)=\left\{e_{2,3}, e_{4,1}\right\}$, and it follows from Corollary 4.1 that $0.88445 \leq \omega_{0}(B) \leq 1.71898$. It follows from Theorem 4.1 that we obtain the accurate result $\omega_{0}(B)=0.97920$. 
Example 5.3 When $A$ is weakly irreducible, $R_{i}(A)=R_{i}(\hat{A}), \forall i \in \mathcal{N}$. Consider the following non-weakly irreducible and nonnegative matrix:

$$
A=\left[\begin{array}{llll}
1 & 1 & 1 & 0 \\
0 & 2 & 1 & 1 \\
0 & 1 & 2 & 1 \\
0 & 0 & 0 & 1
\end{array}\right] .
$$

Obviously, $\rho(A)=3, C(A)=\{\gamma: 2,3,2\}$, and $\Theta_{A}=\{1\}$. In Theorem 3.1, if $r_{A}(\gamma)$ is defined as the real root of $\prod_{i \in \gamma}\left(x-a_{i i}\right)=\prod_{i \in \gamma} R_{i}(A)$, which is larger than $\max _{i \in \gamma}\left\{a_{i i}\right\}$, then $r_{A}(\gamma)=$ 4, and, moreover, $m_{c}^{r}(A)=\max \left\{\min _{\gamma \in C(A)} r_{A}(\gamma), \max \Theta_{A}\right\}=4=\max \left\{\max _{\gamma \in C(A)} r_{A}(\gamma)\right.$, $\left.\max \Theta_{A}\right\}=M_{c}^{r}(A)$. According to Theorem 3.1, obviously it is false. Likewise, if $r_{A}(i, j), l_{A}(\gamma)$, $l_{A}(i, j)$ in Corollary 3.1, Theorem 4.1 and Corollary 4.1 are directly defined by $R_{i}(A)$; mistakes also happen, which will not be discussed in detail.

\section{Competing interests}

The authors declare that they have no competing interests.

\section{Authors' contributions}

All authors contributed equally to the writing of this paper. All authors read and approved the final manuscript.

\section{Acknowledgements}

We wish to thank the anonymous referees for their thorough reading and constructive comments.

Received: 9 March 2014 Accepted: 18 November 2014 Published: 01 Dec 2014

\section{References}

1. Berman, A, Plemmons, RJ: Nonnegative Matrices in the Mathematical Sciences. SIAM, New York (1994)

2. Frobenius, GF: Über Matrizen aus nicht negativen Elementen. Königliche Akademie der Wissenschaften, Berlin (1912)

3. Varga, RS: Geršgorin and His Circles. Springer, New York (2000)

4. Brauer, A, Gentry, IC: Bounds for the greatest characteristic root of an irreducible nonnegative matrix. Linear Algebra Appl. 8, 105-107 (1974)

5. Tong, WT: On the distributions of eigenvalues for some classes of matrices. Acta Math. Sin. 20(4), $272-275$ (1977)

6. Zhang, JJ: On the distributions of eigenvalues for conjugate diagonal dominant matrices. Acta Math. Sin. 23(4), 544-546 (1980)

7. Brualdi, RA: Matrices, eigenvalues, and directed graphs. Linear Multilinear Algebra 11, 143-165 (1982)

8. Zhang, X, Gu, DH: A note on A. Brauer's theorem. Linear Algebra Appl. 196, 163-174 (1994)

9. Li, LL: A simplified Brauer's theorem on matrix eigenvalues. Appl. Math. J. Chin. Univ. Ser. B 14(3), $259-264$ (1999)

10. Kolotilina, YL: Generalizations of the Ostrowski-Brauer theorem. Linear Algebra Appl. 364, 65-80 (2003)

11. Du, YH: An improvement of Brauer's theorem and Shemesh's theorem. Acta Math. Appl. Sin. 27(1), 1-11 (2004)

10.1186/1029-242X-2014-477

Cite this article as: Wang et al.: Estimations for spectral radius of nonnegative matrices and the smallest eigenvalue of M-matrices. Journal of Inequalities and Applications 2014, 2014:477

\section{Submit your manuscript to a SpringerOpen ${ }^{\ominus}$ journal and benefit from:}

- Convenient online submission

Rigorous peer review

- Immediate publication on acceptance

- Open access: articles freely available online

- High visibility within the field

- Retaining the copyright to your article 